\title{
SERRE CLASSES FOR TOPOSES
}

\section{Adelman and P.T. Johnstone}

\begin{abstract}
We prove first that a logical fraction functor from a topos to a topos must be a filter-power functor, then we prove that such functors can have adjoints only when the filter is principal. Finally we refine this so that we are able to prove that the filter-power of a Grothendieck topos is Grothendieck if and only if the filter is principal.
\end{abstract}

\section{Introduction}

If $C$ is a category and $\sum$ a class of morphisms of $C$, it is well known [4] that we may construct a category of fractions $c\left[\Sigma^{-1}\right]$ and a functor $P_{\Sigma}: \mathcal{C} \rightarrow \mathrm{C}\left[\Sigma^{-1}\right]$ which is universal amongst functors $T: \mathcal{C} \rightarrow D$ sending all morphisms in $\Sigma$ to isomorphisms. If $C$ has some categorical structure, it is of interest to characterize those classes $\Sigma$ for which $\mathcal{C}\left[\Sigma^{-1}\right]$ inherits the same kind of structure and $P_{\Sigma}$ preserves it: a wellknown example is the structure of finite limits, for which the corresponding condition on $\Sigma$ is that it should admit a calculus of right fractions $[4$, I 3$]$.

Again, if $A$ is an abelian category, the fractions maps $P_{\Sigma}: A \rightarrow A\left[\Sigma^{-1}\right]$ for which $A\left[\Sigma^{-1}\right]$ is abelian and $P_{\Sigma}$ is exact correspond to Serre classes in A [8], [5]; recall that a Serre class is a class $C$ of objects in $A$ such that if $0 \rightarrow A^{\prime} \rightarrow A \rightarrow A^{\prime \prime} \rightarrow 0$ is an exact sequence in $A$, then $A \in C$ if and only if both $A^{\prime}$ and $A^{\prime \prime}$ are in $C$. The Received 18 August 1981. 
corresponding $\Sigma$ consists of all those $f: A \rightarrow B$ such that both Ker $f$ and Coker $f$ are in $C$; we recover $C$ from $\Sigma$ as the class of all objects $A$ such that $P_{\Sigma}(A) \cong 0$.

In this paper we investigate the analogous problem for (elementary) toposes. We shall show that, for a topos $E$, the fractions maps $P_{\Sigma}: E \rightarrow E\left[\Sigma^{-1}\right]$ for which $E\left[\Sigma^{-1}\right]$ is a topos and $P_{\Sigma}$ is logical correspond to filters of subobjects of 1 in $E$; that is, they are the fizterpowers of $E$ as defined in [7, 9.43] or [1].

In the abelian case, one is particularly interested in the Serre classes which give rise to localizations of $A$, that is, fractions maps $P_{\Sigma}: A \rightarrow A\left[\Sigma^{-1}\right]$ having ( full and faithful) right adjoints. In the topos case, we shall see that the "localizing" filters are just the principal ones, and that the corresponding localizations of $E$ are the categories $E / U$, where $U$ is a subobject of 1 . (Indeed, for a cocomplete topos $E$ we shall prove the stronger result that if a filterpower $E / F$ is cocomplete then already $F$ must be principal.)

However, for toposes we have a more general (and more interesting) notion of localization than the above, namely the notion of sheaf subtopos. Because sheaf reflectors are merely exact and not normally logical, this suggests that we should weaken the conditions on our original problem by requiring only that the functor $P_{\Sigma}$ be exact (while still, of course, demanding that $E\left[\Sigma^{-1}\right]$ be a topos). Unfortunately we have been unable to characterize the classes $\Sigma$ which give rise to such fractions maps, though we shall make a few remarks about the problem at the end of section 2.

A few words are in order about our conventions regarding set theory. Although our main Theorems 2.1 and 2.3 are completely elementary, elsewhere we shall need to restrict ourselves to toposes which are locally smalz; that is, have hom-functors taking values in some fixed topos set of constant sets. As usual, we say a topos $E$ is defined over Set if there is a geometric morphism $E \rightarrow$ Set; it is well known that this happens if and only if $E$ is locally small and has small copowers [7, 4.41]. By a subobject of $X$ in a topos $E$ we mean an isomorphism class of monomorphisms into $X$, though we shall commonly fail to distinguish 
notationally between a subobject of $X$ and a monomorphism which represents it. We write $\operatorname{Sub}(X)$ for the lattice of subobjects of $X$; note that this is small if $E$ is locally small.

\section{The axis of a morphism}

THEOREM 1.1. Let $E$ be a topos and let $f: X \rightarrow Y$ be a morphism in $E$. Then the class

$$
S(f)=\{U \in \operatorname{Sub}(I) \mid U \times f: U \times X \rightarrow U \times Y \text { is an isomorphism }\}
$$

has a greatest member.

Proof. Suppose first that $f$ is a monomorphism. Since the square

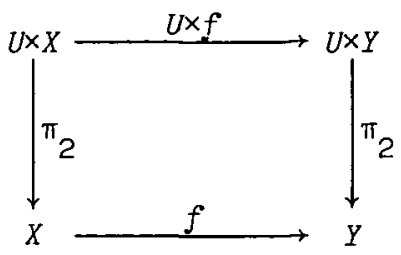

is a pullback, it is clear that $U \in S(f)$ if and only if $U \times Y \leq f$ in $\operatorname{Sub}(Y)$, or equivalently $U \leq \forall_{Y}(f)$ in $\operatorname{Sub}(1)$. So $\forall_{Y}(f)$ is the required greatest element of $S(f)$. In the general case, form the diagram

$$
X>\stackrel{h}{\rightarrow} K \underset{b}{\stackrel{a}{\longrightarrow}} x \underset{q \gamma_{I} \underset{g}{\longrightarrow}}{\stackrel{f}{\longrightarrow}}
$$

where $(a, b)$ is the kernel-pair of $f,(q, g)$ its image factorization and $h$ the factorization of the diagonal $X \rightarrow X \times X$ through $K$. Since $q$ is the coequalizer of $(a, b)$ and $U \times(-)$ is an exact functor $E \rightarrow E / U$, it is clear that we have

$$
U \times f \text { iso } \Leftrightarrow U \times g \text { and } U \times q \text { iso } \Leftrightarrow U \times g \text { and } U \times h \text { iso . }
$$

But $g$ and $h$ are both mono, so by the first part the greatest member of $S(f)$ is $\forall_{Y}(g) \cap \forall_{K}(h)$.

DEFINITION 1.2. Let $f$ be a morphism in a topos. We call the greatest member of $S(f)$ the axis of $f$ and denote it by $A(f)$.

Since the construction of $A(f)$ involves only finite limits, image factorizations and universal quantification, it is clear that we have 
PROPOSITION 1.3. Let $F: E \rightarrow E^{\prime}$ be a functor between toposes preserving finite limits, images and universal quantification (for example a logical fronctor). Then for any morphism $f$ of $E$, we have $F(A(f))=A(F(f))$.

DEFINITION 1.4. Let $F: E+E^{\prime}$ be a left exact functor between finitely complete categories. We define the kernel of $F$, $\operatorname{Ker} F$, to be the class of all subobjects $U$ of $I$ in $E$ such that $F(U) \cong 1$.

PROPOSITION 1.5. Let $F: E \rightarrow E^{\prime}$ be a functor between toposes satisfying the hypotheses of Proposition 1.3. Then

(i) $F(f)$ is an isomorphism if and only if $A(f) \in \operatorname{Ker} F$,

(ii) $F$ is conservative (that is reflects isomorphisms) if and only if $\operatorname{Ker} F=\{1\}$.

Proof. (i) Clearly $F(f)$ is an isomorphism if and only if $A(F(f)) \cong 1$, so this is immediate from Proposition 1.3 and the definition of $\operatorname{Ker} F$.

(ii) follows immediately from (i).

\section{Filters as Serre classes}

Let $E$ be a topos. By a filter on $E$ we mean a filter of subobjects of $l$, that is a collection $F$ of subobjects of 1 such that $1 \in F$ and $(U \cap V) \in F$ if and only if both $U$ and $V$ are in $F$. It is clear that if $F$ is any left exact functor defined on $E, \operatorname{Ker} F$ is a filter on $E$.

THEOREM 2.1. Let $E$ be a topos, and $\Sigma$ a saturated family of morphisms of $E$. Then $E\left[\Sigma^{-1}\right]$ is a topos with $P_{\Sigma}$ logical if and only if there is a fizter $F$ on $E$ such that $\Sigma=\{f \mid A(f) \in F\}$.

Proof. If $\Sigma$ is of the specified form, then an argument due to Lawvere and Tierney (see $[7,9.44]$ ) shows that $E\left[\Sigma^{-1}\right]$ is a topos and $P_{\Sigma}$ is logical. Conversely, suppose $P_{\Sigma}$ is logical. Since $\Sigma$ is saturated, we have $f \in \Sigma$ if and only if $P_{\Sigma}(f)$ is an isomorphism, but, by Proposition $1.5(i)$, this happens if and only if $A(f) \in \operatorname{Ker} P_{\Sigma}$. So if we 
define $F=\operatorname{Ker} P_{\Sigma}$ we have the required description of $\Sigma$.

When $\Sigma$ is induced by a filter $F$ as above, we shall write $E / F$ in place of $E\left[\Sigma^{-1}\right]$, and call it the fitterpower of $E$ relative to $F$. We shall find it convenient to use the following explicit description of $E / F$, given in [1]: its objects are those of $E$, and morphisms are given by

$$
E / F(X, Y)=\operatorname{colim}_{U \in F} E(U \times X, Y)
$$

The conditions which we imposed on the functor $F$ in Proposition 1.5 are weaker than requiring it to be logical; so we obtain the following immediate consequence of the proof of Theorem 2.1.

COROLLARY 2.2. Let $P_{\Sigma}: E \rightarrow E\left[\Sigma^{-1}\right]$ be an exact fractions map between toposes. Then $P_{\Sigma}$ is logical provided it preserves universal quantification.

In the case when $P_{\Sigma}$ has a right adjoint (so that it is the inverse image of a geometric inclusion $\left.E\left[\Sigma^{-1}\right] \rightarrow E\right)$, this result is already known (cf. $[7,3.55])$.

From the description of $E / F$ given above, it is clear that there is no "saturation problem" for filters; that is, $F$ consists precisely of those subobjects of 1 in $E$ whose image in $E / F$ is isomorphic to 1 . For if $l \cong P_{\Sigma}(U)$ in $E / F$, this isomorphism must be represented by a map $V \rightarrow U$ in $E$ with $V \in F$; then $V \subseteq U$ in $\operatorname{Sub}(1)$, and so $U \in F$.

If $F$ is a principal filter

$$
(U)=\{V \in \operatorname{Sub}(1) \mid U \subseteq V\}
$$

for some $U \in \operatorname{Sub}(1)$, then $E / F$ is equivalent to $E / U$; more precisely, the unique factorization through $E \rightarrow E / F$ of the functor $U \times(-): E \rightarrow E / U$ is an equivalence. In this case, therefore, the canonical functor $P: E \rightarrow E / F$ has both left and right adjoints. Our next result says that either of these conditions is sufficient to characterize the filterpowers arising from principal filters.

THEOREM 2.3. Let $F$ be a filter on a topos $E$. The following 
conditions are equivalent:

(i) $F$ is principal;

(ii) $P: E \rightarrow E / F$ is equivalent to $U \times(-): E \rightarrow E / U$ for some $U \in \operatorname{Sub}(1) ;$

(iii) $P: E \rightarrow E / F$ has a right adjoint;

(iv) $P: E \rightarrow E / F$ has a left adjoint.

Proof. The implication ( $i$ ) implies ( $i i)$ follows from the remarks above; (ii) implies (iii) follows from cartesian closedness of $E$ [2, 1.34]; and (iii) implies ( $i v$ ) from the fact that $P$ is logical [6]. So it suffices to show that (iv) implies ( $i$ ). Let $L$ be left adjoint to $P$, and consider the image factorization

$$
L P(1) \rightarrow U>1
$$

of the counit map $\varepsilon: L P(1) \rightarrow 1$. Since $P(\varepsilon)$ is (split) epi, we have $P(U) \cong I$ and hence $U \in F$. Now for any $V \in \operatorname{Sub}(I)$ we have

$$
E(L P(1), V) \cong E / F(P(1), P(V))
$$

and hence

$$
\begin{aligned}
U \subseteq V & \Leftrightarrow \text { there exists a map } L P(1) \rightarrow V \text { in } E \\
& \Leftrightarrow \text { there exists a map } 1=P(1) \rightarrow P(V) \text { in } E / F \\
& \Leftrightarrow P(V) \cong 1 \\
& \Leftrightarrow V \in F .
\end{aligned}
$$

So $F$ is the principal filter generated by $U$.

Theorems 2.1 and 2.3 tell us that the "Serre classes" which correspond to fractions maps in the category of toposes and logical functors are just the filters of subobjects of 1 , and that the "localizing Serre classes" are principal filters. Because of this latter result, the theory of localizations in this category will not be as rich as it is for abelian categories and exact functors. It would therefore be of interest to examine these questions in the larger category of toposes and exact functors; for then the localizing Serre classes in a topos $E$ would correspond to reflective subcategories of $E$ with exact reflector, which by the "little Giraud theorem" [7, 4.15 (i)] correspond to Lawvere-Tierney topologies in $E$. 
However, it is less clear how the (non-localizing) Serre classes should be described in this case. By the argument of the second part of the proof of Theorem 1.1, the class of all morphisms inverted by an exact functor $F: E \rightarrow E^{\prime}$ is determined as soon as one knows that class $\sum$ of monomorphisms inverted by $F$. This class has (at least) the following properties :

(a) a composite $f g$ of two monomorphisms is in $\Sigma$ if and only if both $f$ and $g$ are in $\Sigma$;

(b) pullbacks and pushouts of morphisms in $\Sigma$ are in $\Sigma$;

(c) if the pullback of $f$ along some epimorphism is in $\Sigma$, then $f \in \Sigma$.

If we add the hypothesis that $\Sigma$ is representable by a subobject $J$ of $\Omega$ (that is that it consists of all monos whose classifying maps factor through $J$ ), then it follows from $[7,3.18]$ that $\Sigma$ is a localizing serre class, and in this case the category of fractions $E\left[\Sigma^{-1}\right]$ is a topos $[7$, 3.46]. However, for a general $\Sigma$ satisfying (a)-(c) above, there seems to be no reason why $E\left[\Sigma^{-1}\right]$ should be a topos - though we do not know of a counterexample.

\section{Cocompleteness of filterpowers}

We begin this section with a result which (for cocomplete toposes) refines the equivalence between $(i)$ and (iii) of Theorem 2.3. Let $k$ be an infinite cardinal; we recall that a filter $F$ is said to be $K-$ complete if every subset of $F$ of cardinality $K$ has a lower bound in $F$.

THEOREM 3.1. Let $E$ be a topos in which the $k$-fold copower of 1 exists, $F$ a filter on $E$. Then $F$ is $K$-complete if and only if the canonical functor $P: E \rightarrow E / F$ preserves coproducts of cardinality $K$.

Proof. Suppose $F$ is $k$-complete, and let $\left(x_{\alpha} \mid \alpha \in A\right)$ be a family of objects of cardinality $\kappa$ having a coproduct $\frac{1}{\alpha} x_{\alpha}$ in $E$. We shall show directly that $\frac{1}{\alpha} x_{\alpha}$ is the coproduct of the $x_{\alpha}$ in $E / F$. Let $\left(f_{\alpha}: X_{\alpha}+Y \mid \alpha \in A\right)$ be a family of maps in $E / F$; we can represent them 
by maps $g_{\alpha}: U_{\alpha} \times X_{\alpha} \rightarrow Y$ in $E$, where $U_{\alpha} \in F$ for each $\alpha$. Let $U$ be a lower bound for the $U_{\alpha}$ in $F$; then the composites

$$
U \times x_{\alpha} \longrightarrow U_{\alpha} \times x_{\alpha} \stackrel{g_{\alpha}}{\longrightarrow} y
$$

can be combined to form a map $U \times \frac{1}{\alpha} x_{\alpha} \cong \frac{1}{\alpha}\left(U \times X_{\alpha}\right) \rightarrow Y$ in $E$. But this represents a map $\frac{1}{\alpha} X_{\alpha} \rightarrow Y$ in $E / F$, which is clearly a factorization of the family $\left(f_{\alpha}\right)$ through the maps $P\left(x_{\alpha}+\frac{\perp}{\alpha} x_{\alpha}\right)$. The uniqueness of this factorization is proved by a similar argument; so $\frac{1}{\alpha} X_{\alpha}$ is a coproduct in $E / F$.

Conversely, suppose $P$ preserves coproducts of cardinality $K$, and let $\left(U_{\alpha} \mid \alpha \in A\right)$ be a family of elements of $F$ of cardinality $k$. Since each $U_{\alpha}$ is a subobject of 1 , the coproduct $\frac{1}{\alpha} U_{\alpha}$ exists in $E$; moreover, the inclusion $\frac{1}{\alpha} U_{\alpha} \rightarrow \frac{1}{\alpha} 1$ is sent by $P$ to an isomorphism in $E / F$, and so its axis must be in $F$. But it is easy to see that the axis of this map must be a lower bound for the family $\left(U_{\alpha}\right)$ in $\operatorname{Sub}(1)$.

COROLLARY 3.2. Let $E$ be a topos defined over set, F a fizter on $E$. Then $F$ is principal if and only if the canonical functor $P: E \rightarrow E / F$ preserves (small) coproducts.

Proof. One direction follows from Theorem 2.3 (iii). Conversely, suppose $P$ preserves coproducts. Applying Theorem 3.1 with $K=$ card $F$ (which is possible since $E$ is locally small and hence $F$ is small), we deduce that the family of all elements of $F$ has a lower bound in $F$; that is, $F$ has a least member.

It is natural to ask whether a filterpower $E / F$ might have infinite coproducts in some "nonstandard" way; that is without their being preserved by $P: E \rightarrow E / F$. We devote the rest of this section to proving that the answer is no, at least provided we assume the axiom of choice in Set . First we need a "standard form" for copowers of 1 in $E / F$. 
LEMMA 3.3. Let $E$ be a topos with small copowers, $F$ a filter on $E$ and $A$ a small set. Suppose an $A$-indexed copower of 1 exists in $E / F$. Then it may be taken to be (the image under $P$ of) an A-indexed coproduct of members of $F$ in $E$.

Proof. Let $X$ be an $A$-indexed copower of 1 in $E / F$. For each $\alpha \in A$, the ath coproduct inclusion $1 \rightarrow X$ in $E / F$ may be represented by a map $U_{\alpha} \rightarrow X$ in $E$, for some $U_{\alpha} \in F$. These maps may be combined into a single map $f: \frac{\perp_{\alpha}}{U_{\alpha}} U_{\alpha}$ in $E$ (we reserve the $\perp$ symbol for coproducts in $E$ ). But since $X$ is a coproduct in $E / F$ of the objects $P\left(U_{\alpha}\right)$, it follows that $P(f)$ must be split epi in $E / F$; that is, there is a map $g: V \times X \rightarrow \frac{1}{\alpha} U_{\alpha}$ (for some $V \in F$ ) such that the composite $f g$ represents the identity map $X \rightarrow X$ in $E / F$ (and hence, if we take $V$ small enough, we actually have $\left.f g=\pi_{2}: V \times X \rightarrow X\right)$. Now $g$ is mono (since $\pi_{2}$ is), and hence if we pull back the coproduct decomposition of $\frac{\perp}{\alpha} U_{\alpha}$ along it we obtain a decomposition $V \times X \cong \perp_{\alpha} V_{\alpha}$, for some $V_{\alpha} \subseteq U_{\alpha}$ in $\operatorname{Sub}(1)$. But $V \times X \cong X$ in $E / F$; so it remains to show that the $V_{\alpha}$ are all in $F$.

From the definition of $g$, it is clear that the composite

$$
v \times u_{\alpha} \stackrel{V \times f_{\alpha}}{\longrightarrow} V \times x \stackrel{g}{\longrightarrow} \frac{1}{\alpha} U_{\alpha}
$$

represents the same map $1 \rightarrow \frac{1}{\alpha} U_{\alpha}$ in $E / F$ as the $\alpha$ th coproduct inclusion $U_{\alpha} \rightarrow \frac{1}{\alpha} U_{\alpha} ;$ so there is a $W_{\alpha}$ in $F$ such that

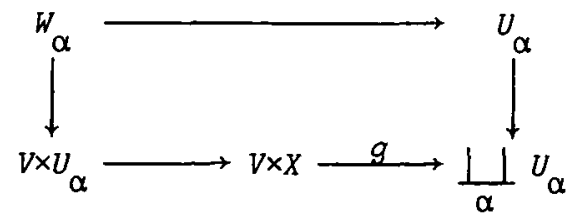

commutes. But since $V_{\alpha}$ is defined to be the pullback of $U_{\alpha}$ along $g$, it follows that $W_{\alpha} \subseteq V_{\alpha}$ and hence $v_{\alpha} \in F$. 
THEOREM 3.4. Let $E$ be a topos defined over Set, and $F$ a filter on $E$. Then $F$ is principal if and only if the filterpower $E / F$ is defined over Set.

Proof. If $F$ is principal, then $E / F$ has small copowers by Corollary 3.2, and it clearly inherits local smallness from $E$. So suppose $F$ is not principal, and assume that an $F$-indexed copower of 1 , say $X$, exists in $E / F$. By Lemma 3.3, we may assume $X=\frac{1}{U \in F} \alpha(U)$ for some function $\alpha: F \rightarrow F$. Since $F$ is not principal, we can choose $B: F \rightarrow F$ such that $U \cap \alpha(U) \nsubseteq \beta(U)$ for all $U$; let $Y$ be the coproduct $\frac{1}{U \in F} \dot{B}(U)$ in $E$. The coproduct inclusions in $Y$ represent maps $1 \rightarrow Y$ in $E / F$, and so induce a map $X \rightarrow Y$ in $E / F$, which can be represented by a map $f: V \times X \rightarrow Y$ in $E$ for some $V \in F$. Restricting this map to the $U$ th factor of the coproduct $X$ gives us a map $f_{U}: V \cap \alpha(U) \rightarrow Y$ in $E$, which represents the same map $1 \rightarrow Y$ in $E / F$ as the Uth coproduct inclusion $\beta(U) \rightarrow \frac{1}{U} \beta(U)=y$. So if we define

$$
\gamma(U)=f_{U}^{-1}(\beta(U)), \delta(U)=f_{U}^{-1}\left(\bigsqcup_{W \neq U} \beta(W)\right),
$$

then we have $\gamma(U) \in F, \quad \gamma(U) \subseteq B(U)$ and $\gamma(U) \perp \delta(U) \cong V \cap \alpha(U)$.

Now define maps $g_{1}, g_{2}: V \times X \rightarrow Y{ }_{\perp} Y$ in $E$ by setting $g_{1}=v_{1} f$ (where $v_{1}$ is the first coproduct inclusion), and demanding that $g_{2}$ should agree with $\nu_{1} f$ and $\nu_{2} f$ repsectively when restricted to the complementary subobjects $\frac{\perp_{U}}{U} \gamma(U)$ and $\frac{\perp_{U}}{U} \delta(U)$ of its domain. Since $g_{1}$ and $g_{2}$ agree on each $\gamma(U)$, it is clear that their composites with the Uth coproduct inclusion represent the same map $1 \rightarrow Y \Perp y$ in $E / F$. So by uniqueness of maps out of coproducts, $g_{1}$ and $g_{2}$ represent the same map $X+Y \perp Y$ in $E / F$; hence there must exist $W \subseteq V$ in $F$ such that $g_{1}$ and $g_{2}$ agree (in $\left.E !\right)$ when restricted to $W \times X$. But since $g_{1}$ and $g_{2}$ disagree on every $\delta(U)$, it follows that we must have $W \cap \delta(U) \cong 0$ for all $U$. Now $V \cap \alpha(W) \cong \gamma(W) \perp \delta(W)$, and so 


$$
W \cap \alpha(W) \subseteq \gamma(W) \perp(W \cap \delta(W)) \cong \gamma(W) \subseteq \beta(W),
$$

contradicting the definition of $B$. Thus if $F$ is non-principal, the $F$-indexed copower of 1 cannot exist in $E / F$.

It is easy to see that the property of possessing a small set of generators is inherited by $E / F$ from $E$; and so we may if we wish replace the phrase "defined over Set "by. "Grothendieck" in the statement of Theorem 3.4 .

\section{The filterpower factorization}

Let Log denote the category of locally small toposes and logical functors between them, and let Heyt denote the category of small Heyting algebras and Heyting algebra homomorphisms. Then there is a functor

$$
S: \log \rightarrow \text { Heyt }
$$

which sends a topos to its lattice of subobjects of 1 , and a logical functor to its restriction to subobjects of $I$. It follows at once from Proposition 1.5 ( $i i$ ) that a logical functor $F$ is conservative if and only if $S(F)$ is a monomorphism; equivalently, if $M$ denotes the class of monos in Heyt, then $S^{-1}(M)$ is the class of conservative logical functors.

Now there is a factorization system $\left(M^{\uparrow}, M\right)$ on Heyt, where $M^{\uparrow}$ is the class of surjective homomorphisms (that is regular epimorphisms). Also, the functor $S$ has a left adjoint $L:$ Heyt $\rightarrow \log$. In this situation, a theorem of Freyd and Kelly [3] tells us that $S^{-1}(M)^{\uparrow}$ is generated by $L\left(M^{\uparrow}\right)$. Unfortunately the functor $L$ is not easy to describe explicitly; however, the use of filterpowers enables us to give an alternative description of $S^{-1}(M)^{\uparrow}$.

We shall call a logical functor $F: E \rightarrow E^{\prime}$ a filterpower functor if there is a filter $F$ on $E$ and an isomorphism (under $E$ ) between $E^{\prime}$ and $E / F$. (It would perhaps be more natural to replace "isomorphism" by "equivalence" in this definition; this change would not introduce any serious extra difficulties, but it would force us to refer to the 2-categorical structure of $\log$ in the statement of the theorem which follows, and so for the sake of brevity we shall not make it.) 
THEOREM 4.1. The classes \{filterpower functors\} and \{conservative functors\} form a factorization system on Log. Moreover, the members of the first class are epimorphisms in $\log$, and $\log$ is cowe Zz-powered with respect to them.

Proof. Given any logical functor $F: E \rightarrow E^{\prime}$, let $F=\operatorname{Ker} F$. Then by Proposition 1.5 (i) we have a factorization

$$
E \stackrel{P}{\longrightarrow} E / F \stackrel{G}{\longrightarrow} E^{\prime}
$$

of $F$, in which $\operatorname{Ker} G=P(\operatorname{Ker} F)=\{I\}$ and so $G$ is conservative. Now suppose given a commutative diagram

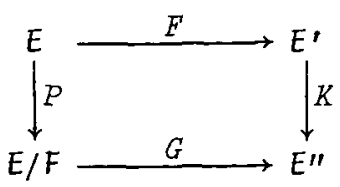

where $K$ is conservative. Then for any morphism $f$ in $E$ we have

$$
P(f) \text { iso } \Rightarrow G P(f)=K F(f) \text { iso } \Rightarrow F(f) \text { iso, }
$$

and so there is a unique $L: E / F \rightarrow E^{\prime}$ such that $L P=F$. It is clear from the universal mapping property of fractions maps that they are epis in Cat (and hence in $\log$ ), and so we also have $K L=G$. Finally, the isomorphism classes of filterpower functors with a fixed domain $E$ are parametrized by the filters on $E$, which in turn are parametrized by a subset of $2^{E(I, \Omega)}$. So $\log$ is co-well-powered.

By a standard Adjoint-Functor-Theorem argument, we may obtain the following consequence of Theorem 4.1.

COROLLARY 4.2. Let $P$ be a property of toposes which is inherited by products and reflected by conservative logical functors (for example, Booleanness). Then the full subcategory of toposes with $P$ is reflective in $\log$.

\section{References}

[1] M. Adelman, "On the forcing construction", Report of the Sydney Category Seminar (1978). 
[2] Peter Freyd, "Aspects of topoi", Bulz. Austral. Math. Soc. 7 (1972), $1-76$.

[3] P.J. Freyd and G.M. Kelly, "Categories of continuous functors, I", J. Pure Appl. AZgebra 2 (1972), 169-191.

[4] P. Gabriel and M. Zisman, Calculus of fractions and homotopy theory (Ergebnisse der Mathematik und ihrer Grenzgebiete, 35. SpringerVerlag, Berlin, Heidelberg, New York, 1967).

[5] Alexandre Grothendieck, "Sur quelques points d'algèbre homologique", Tôhoku Math. J. (2) 9 (1957), 119-221.

[6] P.T. Johnstone, "Adjoint lifting theorems for categories of algebras", Bul2. London Math. Soc. 7 (1975), 294-297.

[1] P.T. Johnstone, Topos theory (London Mathematical Society Monographs, 10. Academic Press [Harcourt Brace Jovanich], London, New York, San Franscisco, 1977).

[8] Jean-Pierre Serre, "Groupes d'homotopie et classes de groupes abéliens", Ann. of Math. 58 (1953), 258-294.

School of Mathematics and Physics,

Macquarie University,

North Ryde,

New South Wales 2113,

Australia;

Department of Pure Mathematics,

University of Cambridge,

Cambridge CB2 ISB,

England. 